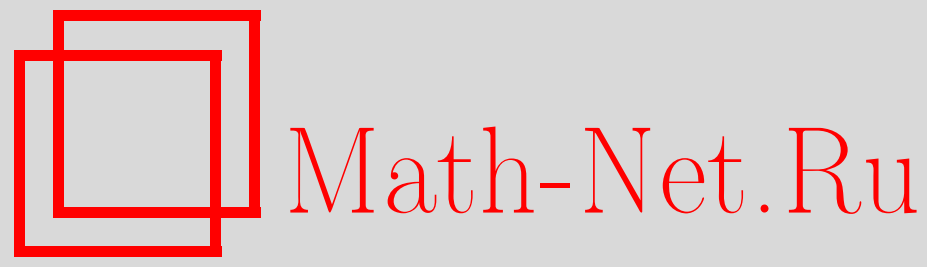

Ю. И. Манин, Закон Ципфа и вероятностные распределения Левина, Функи. анализ и его прил., 2014, том 48, выпуск 2, 51-66

DOI: https://doi.org/10.4213/faa3141

Использование Общероссийского математического портала MathNet.Ru подразумевает, что вы прочитали и согласны с пользовательским соглашением

http://www . mathnet.ru/rus/agreement

Параметры загрузки:

IP : 54.80 .97 .219

26 апреля 2023 г., 16:09:46

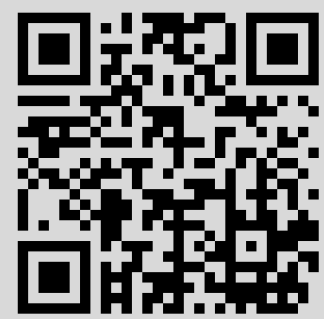




\title{
Закон Ципфа и вероятностные распределения Левина
}

\author{
(c) 2014. Ю. И. МАнин
}

Памяти Израиля Моисеевича Гельфанда

\begin{abstract}
Закон Ципфа в его классической форме описывает эмпирическое вероятностное распределение, которому подчиняются частоты использования слов в языке. Как недавно заметил Теренс Тао, этот закон до сих пор не имеет убедительного и удовлетворительного математического объяснения.

В этой статье я высказываю предположение, что по крайней мере в некоторых ситуациях закон Ципфа можно получить как частный случай априорного распределения, введенного и изученного Л. Левиным. При этом ципфовское упорядочение, соответствующее убыванию вероятностей, возникает как упорядочение по возрастанию колмогоровской сложности.

Один из аргументов в защиту этого тезиса связан с интерпретацией асимптотических границ для кодов, исправляющих ошибки, в терминах фазового перехода, предложенной недавно Ю. Маниным и М. Марколли. В соответствующей статсумме колмогоровская сложность кода играет роль его энергии.
\end{abstract}

\section{Введение и основные результаты}

0.1. Закон Ципфа. В своей исходной форме закон Ципфа представляет собой следующее эмпирическое наблюдение ([24], [25]): если все слова $w_{k}$ произвольного языка упорядочить по убыванию частоты, с которой они встречаются в некотором репрезентативном корпусе текстов, то частота $p_{k}$ слова $w_{k}$ будет (приблизительно) обратно пропорциональна его порядковому номеру (называемому рангом) $k$; см., например, рис. 1 в работе [11], где приведены данные для словаря, содержащего $4 \cdot 10^{7}$ слов русского языка.

Многочисленные другие примеры проявлений данного степенного закона с показателем -1 для различных наборов статистических данных приведены в работе [17] и цитирующейся в ней литературе.

В избытке имеются и теоретические модели распределения Ципфа. Сам Ципф в основополагающих работах [25], [24] высказывал мысль, что его распределение «минимизирует усилия». Мандельброт [10] описал точную математическую основу для построения модели закона Ципфа. А именно, если мы введем «цену» (создания, использования и т. п.) слова ранга $k$, обозначив ее через $C_{k}$, то распределение частот $p_{k} \sim 2^{-h^{-1} C_{k}}$ минимизирует отношение $h=C / H$, где $C:=\sum_{k} p_{k} C_{k}-$ средняя цена одного слова, а $H:=-\sum_{k} p_{k} \log _{2} p_{k}-$ средняя энтропия; см. [12].

Отсюда мы получаем степенной закон, если $C_{k} \sim \log k$. Дополнительный вопрос о том, чем выделен показатель степени -1 , нужно исследовать отдельно. Одна из возможностей для этого рассмотрена в [17, разд. III]. В настоящей работе мы предлагаем использовать другую математическую базу (см. следующий раздел). 
Во всех подобных обсуждениях более или менее неявно предполагается, что эмпирически наблюдаемые распределения относятся к фрагментам потенциально счетного бесконечного множества объектов. Я также использую это предположение и работаю с «конструктивным миром» подобного рода; точное определение см. в разд. 1.1 ниже.

0.2. Закон Ципфа на основе сложности. В этой работе я высказываю мысль, что (по крайней мере в некоторых ситуациях) закон Ципфа возникает как следствие одновременного действия двух факторов, (А) и (В).

(А) Упорядочение по рангу совпадает с упорядочением по возрастанию (экспоненииальной) колмогоровской сложности $K(w)$ с точностъю до множителя вида $\exp (O(1))$.

Точнее, чтобы определить сложность $K(x)$ натурального числа $x \in \mathbb{Z}^{+}$, мы выбираем оптимальный по Колмогорову код, который представляет собой частично рекурсивную функцию $u: \mathbb{Z}^{+} \rightarrow \mathbb{Z}^{+}$, и полагаем $K(x)=K_{u}(x):=$ $\min \{y \mid u(y)=x\}$. Другой выбор функции $u$ приводит к изменению значения $K_{u}(\cdot)$ на множитель вида $\exp (O(1))$.

Далее, сложность $K(w)$ элемента $w$ конструктивного мира определяется как сложность его номера в фиксированном структурном упорядочении (см. разд. 1.1 ниже). При изменении этого упорядочения сложность опять изменяется в $\exp (O(1))$ раз.

(В) Вероятностное распределение, приводящее $к$ закону Ципфа (с показателем -1), аппроксимирует максимальное вычислимое снизу распределение Левина; см. [26], [8] и [9].

Если принять тезисы (А) и (B), то закон Ципфа следует из двух фундаментальных свойств колмогоровской сложности:

(a) ранг слова $w$, определенный согласно (A), есть $\exp (O(1)) \cdot K(w)$;

(b) распределение Левина приписывает обгекту $w$ вероятность $\sim K P(w)^{-1}$, где КР - экспонента от префиксной колмогоровской сложности; при этом с точностью до множителей вида $\exp (O(1))$ выполняются соотношения

$$
K(w) \preceq K P(w) \preceq K(w) \cdot \log ^{1+\varepsilon} K(w)
$$

с произвольным $\varepsilon>0$.

Небольшое расхождение между порядками роста функций $K$ и $K P$ - это та причина, по которой с помощью $K$ нельзя построить вероятностное распределение на бесконечном множестве объектов: ряд $\sum_{m} K(m)^{-1}$ расходится. Однако на конечных множествах данных это малое расхождение дополнительно маскируется зависимостью и $K$, и $K P$ от выбора оптимального кода. Поэтому, говоря о законе Ципфа, мы обычно не будем обращать внимания на эту разницу. См. также обсуждение статсуммы для кодов в разд. 0.3 ниже.

0.3. Сложность как усилие. Описанная выше картина согласуется с лозунгом «минимизации усилия» Ципфа, но по-новому интерпретирует понятие усилия: его роль теперь играет логарифм колмогоровской сложности, т. е. длина максимально сжатого описания объекта. Эта длина невычислима, но является точной нижней гранью последовательности вычислимых функций.

Эта интерпретация особенно разумна, если объекты, удовлетворяющие закону Ципфа, не просто наблюдаются, а генерируются. 
На интуитивном уровне описанную картину можно сформулировать так: как только разум отдельного человека или общество в целом находит сжатое описание чего-то, это что-то становится пригодным к употреблению и начинает использоваться чаще, чем другое «что-то», имеющее более длинное описание. Расширенную версию этой метафоры в применении к истории науки можно найти в статье [15].

С точки зрения исходного наблюдения Ципфа, этот принцип описывает то, как разум/мозг создает и использует язык.

0.4. Связь с предыдущими работами. Мне известны две работы, в которых сложность упоминается в связи с законом Ципфа: [22] и [17]. ${ }^{1)}$

(а) Точка зрения авторов статьи [17] в целом близка к нашей, но, грубо говоря, их внимание сосредоточено на большинстве объектов - «почти случайных» объектах, а именно тех, размер которых сравним с колмогоровской сложностью и которые, следовательно, нельзя сжать. Оправданием этому служит принимаемое авторами предположение, что корпус данных, удовлетворяющих закону Ципфа, возникает из последовательности наблюдений за некоторой системой, демонстрирующей стохастическое поведение.

Наше упорядочение, напротив, выводит на передний план те объекты, размер которых может быть велик по сравнению с их сложностью, так как мы представляем себе системы, которые не просто наблюдаются, а генерируются - в том же смысле, в котором тексты на разных языках генерируются, создаются, человеческим мозгом.

Чтобы осознать принципиальную разницу между двумя этими подходами на хорошо понятом математическом примере, можно, следуя работе [16], сравнить их в контексте кодов, исправляющих ошибки. Каждый такой код $C$ (над фиксированным алфавитом) задает точку единичного квадрата на плоскости (скорость передачи, минимальное относительное расстояние Хэмминга). Замыкание множества всех предельных кодовых точек есть область под некоторой непрерывной кривой, которая называется асимптотической гранищей.

Если генерировать коды в порядке возрастания их размера, то большая часть кодовых точек будет образовывать облако, плотно аппроксимирующее так называемую границу Варшамова-Гилберта, которая лежит строго ниже асимптотической границы.

Если же, наоборот, генерировать коды в порядке возрастания их колмогоровской сложности, то кодовые точки будут хорошо аппроксимировать всю область под асимптотической границей; подробности см. в [16]. Более того, распределение Левина очень естественным образом приводит к термодинамической статсумме на множестве кодов и к интерпретации асимптотической границы как кривой фазового перехода: эта статсумма имеет вид $\sum_{C} K(C)^{-s(C)}$, где $s(C)-$ некоторая функция, определенная на кодах и имеющая в качестве параметров аналоги температуры и плотности. Здесь можно заменить $K$ на $K P$ и произвольным образом выбирать оптимальное семейство, задающее сложность: это вообще не изменит вид фазовой кривой/асимптотической границы.

1) Когда этот текст был уже почти полностью написан, появилась еще одна работа, [5], где предлагается по существу та же связь между законом Ципфа и сложностью. Профессор Жан-Поль Делайе любезно обратил на нее мое внимание после того, как моя статья появилась в архиве arXiv.org. 
Величина $\log K(w)$, представляющая собой битовую длину максимально сжатого описания элемента $w$, играет в точности роль энергии, оправдывая наше предложение отождествить ее с «усилием».

Интересно отметить, что исторически прорыв в решении математической проблемы генерации хороших кодов, исправляющих ошибки, произошел в 1980-е годы после открытия алгебро-геометрических кодов Гоппы, т. е. именно после открытия сильно сжатых описаний больших комбинаторных объектов.

Подводя итог, можно сказать, что класс рассматриваемых нами априорных вероятностных распределений качественно отличается от класса распределений, широко используемых в настоящее время в социологическом, а также иногда естественно-научном анализе: см. прекрасный краткий обзор последнего класса в работе Теренса Тао [21], который подчеркивает также, что «у математиков нет вполне удовлетворительного и убедительного объяснения того, откуда берется закон [Ципфа] и почему он столь универсален».

(b) Обратимся теперь к статье [22], в которой Вельдхайзен рассматривает закон Ципфа в необычном контексте, который отсутствовал во времена открытий Колмогорова, Соломонова и Чейтина и который в некотором смысле открывает возможности для использования сложности в промышленных целях. А именно, он изучает реальные библиотеки программного обеспечения и анализирует возможные выгоды от его повторного использования. Это метафора человеческой культуры, повседневное существование которой зависит от постоянного повторного использования сокровищ, созданных учеными, поэтами, философами.

С математической точки зрения, повторное использование дает новые средства сжатия: грубо говоря, функция $f$ может иметь очень большую колмогоровскую сложность, но при этом длина библиотечного адреса ее программы может быть короткой; однако лишь это последнее обстоятельство и существенно, если программу можно просто скопировать из библиотеки.

Чтобы построить математическую модель повторного использования и ее ципфовский рельеф в соответствии с идеями, развиваемыми в этой статье, мне необходимо ввести математическое понятие относительной колмогоровской сложности $K(f \mid F)$. Это понятие восходит к самому Колмогорову и хорошо известно в случае, когда $f, F-$ конечные комбинаторные объекты, например строки целых чисел (см. [9]).

В основном тексте статьи я обобщаю это определение на случай библиотеки $F$ программ. Библиотека может содержать даже невычислимые данные, получаемые от оракула; тем самым мы включаем в рассматриваемый контекст вычисления с оракулом.

0.5. Эмпирические подтверждения. Рассмотрим некоторые экспериментальные данные, демонстрирующие зависимость ципфовского ранга от сложности в наиболее естественном контексте - при изучении статистики не всех слов, а лишь числительных, т. е. имен чисел.

Тогда в нашей модели ожидается следующее поведение:

(i) Вероятности появления большинства чисел $n$ - тех, которые имеют максимальную колмогоровскую сложность, - будут сравнимы с $n^{-1}(\log n)^{-1-\varepsilon}$ с малым $\varepsilon$ : «почти все большие числа встречаются с частотой, обратной к их размеру» (на самом деле, с несколько меньшей). 
(ii) Однако числа, которые очень просты по Колмогорову, например $10^{3}$ (тысяча), $10^{6}$ (миллион), $10^{9}$ (миллиард), должны давать острые локальные пики на графике функции $\left(p_{n}\right)$.

Читатель может сравнить эти свойства обсуждаемого класса распределений Левина, которые можно назвать априорными распределениями, с наблюдаемыми частотами порядковых числительных в печатных и устных текстах на нескольких языках, обзор которых приведен в [3, с. 111, рис. 4.4]. (Части графиков Деана и Мелера из книги [3], относящиеся к большим числам, могут вводить в заблуждение, создавая впечатление, что частоты порядковых числительных, скажем, в интервале между $10^{6}$ и $10^{9}$ даются гладкой интерполяцией между частотами самих чисел $10^{6}$ и $10^{9}$, тогда как на самом деле внутри интервала они резко падают. Однако гораздо более подробное обсуждение имеется в работе [4].) В статье [5] также цитируются обширные эмпирические данные, полученные с помощью поисковой системы Google.

С моей точки зрения, наблюдаемое качественное согласие между предлагаемой теорией и экспериментальными данными выглядит убедительно: отдельные умы и общества действительно следуют предсказаниям, даваемым априорными вероятностями. Разумеется, при этом необходимо помнить, что степень сжатия, доступная умам и цивилизациям, может приводить к количественно отличным распределениям на начальных участках колмогоровской вселенной, ибо здесь особенно ярко проявляется зависимость сложности объектов от сложности порождающих их механизмов («культурный код»).

Несомненно, многие примеры эмпирических законов Ципфа не сводятся к рассматриваемому нами источнику сложности. Возможно, обоснование подобной редукции закона Ципфа в случае всех слов требует обращения к нейробиологии: см. Appendix А в версии работы [11], опубликованной в apхиве arXiv . org.

Еще один интересный потенциальный источник закона Ципфа рассмотрен в недавней статье [6]. Ее авторы предположили, что ципфовский ранг объекта - элемента некоторой вселенной - может совпадать с его характеристикой PageRank относительно подходящей направленной сети, связывающей все объекты. Подобный механизм обычно приводит к степенному закону, но не обязательно именно к закону Ципфа.

Так или иначе, именно использование невычислимой степени максимального сжатия в нашей модели распределения Ципфа-Левина может сделать ее прорывной.

\section{6. Фрактальный характер колмогоровской сложности и универ-} сальность закона Ципфа. График логарифмической колмогоровской сложности (и ее префиксных вариантов) целых чисел $k$ выглядит следующим образом: большую часть времени он близко следует графику функции $\log k$, но бесконечно часто падает вниз - ниже любой заданной вычислимой функции: см. [9, с. 103, 105, 178]. Видимая «непрерывность» этого графика отражает тот факт, что число $k+1$ при любом разумном кодировании имеет почти ту же сложность, что и $k$.

Однако подобная картина не передает исключительно богатых свойств самоподобия, которыми обладает сложность. Основное ее фрактальное свойство таково: если упорядочить любое бесконечное разрешимое подмножество в $\mathbb{Z}^{+}$ по возрастанию и ограничить на него граф сложности, то мы увидим тот же самый сложностный профиль, что и для всего множества $\mathbb{Z}^{+}$; на самом деле для 
любой рекурсивной биекции $f$ множества $\mathbb{Z}^{+}$на его подмножество выполняется соотношение $K(f(x))=\exp (O(1)) \cdot K(x)$.

По-видимому, этот источник «фрактализации» может иметь гораздо более широкое значение: см. [18] и близкие работы.

При переходе от сложности к распределению Левина, т. е., по существу, при обращении значений сложности, эти фрактальные свойства сохраняются.

Возможно, именно это свойство отвечает за «универсальность» закона Ципфа, поскольку его можно трактовать как исключительную устойчивость этого закона относительно перехода к различным подвселенным объектов, их вычислимым перенумерациям и т. п. Именно на ней основано предложение, выдвинутое в работе [7], использовать наблюдаемые отклонения от закона Ципфа для паттернов в обширных базах данных для поиска потенциально фальшивых записей при аудите.

Аналогичным образом, случайный шум на устойчивом фоне отвечает за универсальность нормального распределения.

0.7. План статьи. В основном тексте статьи я больше не пытаюсь доказывать, что источником распределения Ципфа может быть сложность. Вместо этого я привожу набросок математического описания сложности в более широком контесте, обеспечивающем его приложимость к ситуациям, рассмотренным B $[22]$.

В 11 я определяю понятие колмогоровской сложности относительно допустимого семейства (частичных) функций. Постулируемые свойства таких допустимых семейств должны отражать наши интуитивные представления о повторном использовании библиотек и/или вычислениях с оракулом.

В $§ 2$ я предлагаю формализацию понятия вычислений, которая (потенциально) приводит к допустимым семействам. Как и предполагалось в [13, гл. IX] здесь оказываются полезными понятия из теории категорий и операд.

Благодарности. О законе Ципфа я впервые узнал из (одной из первых версий) статьи Д. Ю. Манина [11], а область применимости своих собственных конструкций осознал глубже, пытаясь ответить на его вопросы.

Мысль о том, что закон Ципфа, возможно, является отражением распределения Левина, пришла мне в голову после изучения графиков из книги Деана [3]. Профессор Деан любезно прислал мне также оригинальную статью [4]. К. Калюд прочитал несколько версий этой статьи и способствовал лучшему изложению моих аргументов. А. Дёринг исправил несколько опечаток в ранней версии статьи.

Описание множества программ для вычисления (примитивно) рекурсивных функций с помощью операд (ср. [1]) обсуждалось в работе [23], и моя старая электронная переписка с Н. Янофски помогла прояснить мой подход к формализации понятий повторного использования и оракулов. Л. Левин предложил несколько полезных поправок. Всем им я очень благодарен.

Наконец, я посвящаю эту статью памяти И. М. Гельфанда. В статье «Некоторые вопросы исследования движений» (1966), написанной в соавторстве с коллегами В. С. Гурфинкелем, М. Л. Шиком и М. Л. Цетлиным, он выдвинул идею о том, что универсальный принцип метафизики, определяющий структуру физических законов, - «принцип наименьшего действия» - приложим и к исследованию нервной системы: 
«Нам представляется вероятным, что [... индивидуальная иель, преследуемая подсистемой ... - Ю. М.] состоит в уменвшении полного импульса, получаемого подсистемой как с периферии, так и от частей других подсистем и высших уровней нервной системы (приниип наименъшего взаимодействия)».

\section{§1. Допустимые множества частичных функций и относительная сложность}

1.1. Обозначения и соглашения. Здесь мы напомним некоторые основные соглашения, принятые в $[13$, гл. V и IX]. Пусть $X, Y$ - два множества. Чaстичная функиия из $X$ в $Y$ - это пара $(D(f), f)$, где $D(f) \subset X, f: D(f) \rightarrow Y$. Множество $D(f)$ мы называем областъю определения функции $f$ и часто пишем просто $f: X \rightarrow Y$. Если $D(f)=\varnothing$, то $f$ называется пустой функцией. Если $D(f)=X$, мы иногда называем $f$ всюду определенной функцией. Если $X$ - одноэлементное множество, то непустые функции $X \rightarrow Y$ канонически отождествляются с элементами множества $Y$. Из частичных функций очевидным образом можно составлять композиции: $D(g \circ f):=f^{-1}(D(g) \cap \operatorname{Im}(f))$. Таким образом, мы можем рассмотреть категорию, состоящую из (некоторых) множеств и частичных отображений между ними.

Пусть $\mathbb{Z}^{+}$- множество положительных целых чисел. Тогда множество $\left(\mathbb{Z}^{+}\right)^{m}$ при $m \geqslant 1$ можно отождествить с множеством векторов $\left(x_{1}, \ldots, x_{m}\right), x_{i} \in \mathbb{Z}^{+}$. По определению $\left(\mathbb{Z}^{+}\right)^{0}$ - это одноэлементное множество, скажем $\{*\}$. Любая частичная функция $f:\left(\mathbb{Z}^{+}\right)^{m} \rightarrow\left(\mathbb{Z}^{+}\right)^{n}$ будет называться $(m, n)$-функцией. При $m=0$ непустая $(m, n)$-функция может и будет отождествляться с вектором из $\left(\mathbb{Z}^{+}\right)^{n}$.

Пусть $X$ - бесконечное множество. Структура конструктивного мира на $X$ задается множеством биекций $\mathscr{N}_{X}: X \rightarrow \mathbb{Z}^{+}$, называемых структурными нумерациями, таких, что любые две биекции из $\mathscr{N}_{X}$ связаны (всюду определенной) рекурсивной перестановкой множества $\mathbb{Z}^{+}$и, наоборот, при любой композиции структурной нумерации с рекурсивной перестановкой вновь получается структурная нумерация. Явно заданные конечные множества также рассматриваются как конструктивные миры. (Читатель, склонный мыслить в логических терминах, может считать, что все наши основные конструкции осуществляются на первом этаже иерархии фон Неймана.)

На интуитивном уровне можно представлять себе, что множество $X$ состоит из некоторых конечных структур Бурбаки, однозначно описывающихся и кодирующихся конечными строками в конечном алфавите, которые образуют разрешимое множество строк и, следовательно, также допускают естественную нумерацию. Разумеется, любые две такие естественные нумерации должны быть связаны вычислимой биекцией.

Множество морфизмов между двумя конструктивными мирами по определению состоит из тех теоретико-множественных отображений, которые после выбора структурных нумераций превращаются в частично рекурсивные функции. Таким образом, конструктивные миры являются объектами некоторой категории - конструктивной вселенной.

Чтобы формализовать вычисления с оракулом, нам придется расширить множества морфизмов, разрешив частичные отображения, которые могут быть невычислимыми. 
1.2. Допустимые множества функций. Рассмотрим множество $\Phi$ частичных функций $f:\left(\mathbb{Z}^{+}\right)^{m} \rightarrow\left(\mathbb{Z}^{+}\right)^{n}, m, n \geqslant 0$. Мы будем называть $\Phi$ доnyстимым множеством, если оно счетно и удовлетворяет следующим условиям.

(i) Множество $\Phi$ замкнуто относительно композиции и содержит все проекции (отображения, которые забывают некоторые координаты) и вложения (отображения, которые переставляют координаты и/или добавляют несколько постоянных координат).

Любую $(m+1, n)$-функцию можно рассматривать как семейство $(m, n)$-функций $\left(u_{k}\right): u_{k}\left(x_{1}, \ldots, x_{m}\right):=u\left(x_{1}, \ldots, x_{m}, k\right)$. Из условия (i) следует, что для любой функции $u \in \Phi$ и любого $k \in \mathbb{Z}^{+}$функция $u_{k}$ также лежит в $\Phi$. Аналогично, если функция $u\left(x_{1}, \ldots, x_{m}\right)$ лежит в $\Phi$, то функция

$$
U\left(x_{1}, \ldots, x_{m}, x_{m+1}, \ldots, x_{m+n}\right) \equiv u\left(x_{1}, \ldots, x_{m}\right)
$$

также лежит в $\Phi$.

(ii) Для любой парь $(m, n)$ существует такая $(m+1, n)$-функиия $u \in \Phi$, что семейство функций $u_{k}:\left(\mathbb{Z}^{+}\right)^{m} \rightarrow\left(\mathbb{Z}^{+}\right)^{n}$ содержит все $(m, n)$-функции, принадлежащие множеству $\Phi$.

Будем называть функцию $u$, удовлетворяющую этому условию, а также соответствующее семейство $\left(u_{k}\right)$ обильными.

(iii) Пусть $f$ - всюду определенная рекурсивная функиия, образ которой разрешим, и пусть $f$ задает биекиию между $D(f)$ и своим образом. Тогда множество $\Phi$ содержит $и f, u f^{-1}$.

С этого момента буквой $\Phi$ мы всегда обозначаем допустимое семейство.

1.3. Сложность относительно семейства функций. Возьмем $(m+1, n)$ функцию $u \in \Phi$ и рассмотрим ее как семейство $(m, n)$-функций $u_{k}$ (см. выше). Для произвольной $(m, n)$-функции $f \in \Phi$ положим $K_{u}^{\Phi}(f)=\min \left\{k \mid f=u_{k}\right\}$; если такого $k$ не существует, мы полагаем, что правая часть равна $\infty$. Мы будем говорить, что семейство и оптимально по Колмогорову в $\Phi$, если для любой другой $(m+1, n)$-функции $v$ существует такая константа $c_{u, v}$, что $K_{u}^{\Phi}(f) \leqslant$ $c_{u, v} K_{v}^{\Phi}(f)$ для всех $(m, n)$-функций $f \in \Phi$.

1.4. Теорема. (а) Если множество $\Phi$ содержит обильное семейство $(m+$ $1, n)$-функиий, то оно содержит и оптимальное по Колмогорову семейство $(m, n)$-функиий.

(b) Если и и -два оптимальных по Колмогорову семейства $(m, n)$-функu,uน̆, mo

$$
c_{v, u}^{-1} \leqslant K_{u}^{\Phi}(f) / K_{v}^{\Phi}(f) \leqslant c_{u, v} .
$$

Доказательство. Пусть $\theta: \mathbb{Z}^{+} \times \mathbb{Z}^{+} \rightarrow \mathbb{Z}^{+}-$всюду определенная рекурсивная биекция между $\mathbb{Z}^{+} \times \mathbb{Z}^{+}$и разрешимым подмножеством в $\mathbb{Z}^{+}$. Далее, предположим, что $\theta(k, j) \leqslant k \cdot \phi(j)$ для некоторой функции $\phi: \mathbb{Z}^{+} \rightarrow \mathbb{Z}^{+}$. Выберем обильное семейство $(m+1, n)$-функций $U \in \Phi$ и положим

$$
u\left(x_{1}, \ldots, x_{m}, k\right):=U\left(x_{1}, \ldots, x_{m}, \theta^{-1}(k)\right) .
$$

Тогда семейство $u$ обильно и оптимально, причем константа $c_{u, v}$ удовлетворяет следующей оценке:

$$
c_{u, v} \leqslant \phi\left(K_{U}^{\Phi}(v)\right) .
$$


На самом деле достаточно рассмотреть такое семейство $v$, что функция $f$ встречается в $\left(v_{k}\right)$. Тогда

$$
f\left(x_{1}, \ldots, x_{m}\right)=v\left(x_{1}, \ldots, x_{m}, K_{v}^{\Phi}(f)\right)=U\left(x_{1}, \ldots, x_{m}, K_{v}^{\Phi}(f), K_{U}^{\Phi}(v)\right),
$$

откуда следует, что

$$
K_{u}^{\Phi}(f) \leqslant \theta\left(K_{v}^{\Phi}(f), \quad K_{U}^{\Phi}(v)\right) \leqslant K_{v}^{\Phi}(f) \phi\left(K_{U}^{\Phi}(v)\right) .
$$

1.5. Константы, связанные с оценками колмогоровской сложности. В неравенстве (1.1), оценивающем зависимость колмогоровской сложности от выбора кодировки, основную роль играют две величины.

Первая из них - это $K_{U}^{\Phi}(v)$. Эффективное вычисление этой величины зависит от возможности трансляции программы для $v$ в программу, задаваемую семейством $U$. В случае, когда множество $\Phi$ состоит из всех частично рекурсивных функций, такое преобразование возможно, если $U$ обладает некоторым свойством, более сильным, чем обильность: см. работы [19] и [20], где подобные семейства обсуждаются и строятся.

Другая величина - это скорость роста функции $\phi$. Ниже мы покажем, воспроизводя рассуждения из работы [14], как задачу оптимизации функции $\phi$ можно рассматривать в контексте распределений Левина.

1.5.1. Медленно растущие нумерации множества $\left(\mathbb{Z}^{+}\right)^{2}$. Пусть $R=$ $\left(R_{k} \mid k \in \mathbb{Z}^{+}\right)$- последовательность положительных чисел, стремящаяся $\mathrm{k}$ бесконечности с ростом $k$. При $M \in \mathbb{Z}^{+}$положим

$$
V_{R}(M):=\left\{(k, l) \in\left(\mathbb{Z}^{+}\right)^{2} \mid k R_{l} \leqslant M\right\} .
$$

Очевидно, что

$$
\operatorname{card} V_{R}(M) \leqslant \sum_{l=1}^{\infty}\left[\frac{M}{R_{l}}\right]<\infty,
$$

где через $[a]$ обозначена целая часть числа $a$.

Имеем

$$
V_{R}(M) \subset V_{R}(M+1), \quad\left(\mathbb{Z}^{+}\right)^{2}=\bigcup_{M=1}^{\infty} V_{R}(M) .
$$

Следовательно, можно определить биекцию $N_{R}:\left(\mathbb{Z}^{+}\right)^{2} \rightarrow \mathbb{Z}^{+}$таким образом: $N_{R}(k, l)$ есть ранг элемента $(k, l)$ относительно полного упорядочения $<_{R}$ множества $\left(\mathbb{Z}^{+}\right)^{2}$, определенного по индукции следующим правилом: $(i, j)<_{R}(k, l)$ в том и только том случае, когда выполняется одна из следующих возможностей:

(a) $i R_{j}<k R_{l}$;

(b) $i R_{j}=k R_{l}$ и $j<l$.

1.5.2. Предложение. Нумерачия $N_{R}$ корректно определена и обладает следующим свойством: ранг любого элемента множества $V_{R}(M+1) \backslash V_{R}(M)$ строго больше ранга любого элемента множества $V_{R}(M)$. Более того,

(i) если множество $\left\{(q, l) \in \mathbf{Q} \times \mathbb{Z}^{+} \mid q \geqslant R_{l}\right\}$ перечислимо (является образом частично рекурсивной функции), то функиия $N_{R}$ вычислима (общерекурсивна);

(ii) если ряд $\sum_{l=1}^{\infty} R_{l}^{-1}$ сходится и его сумма ограничена константой $c$, то

$$
N_{R}(k, l) \leqslant c\left(k R_{l}+1\right)
$$


(iii) если ряд $\sum_{l=1}^{\infty} R_{l}^{-1}$ расходится $и$

$$
\sum_{l=1}^{M} R_{l}^{-1} \leqslant F(M)
$$

для некоторой возрастающей функиии $F=F_{R}$, то

$$
N_{R}(k, l) \leqslant\left(k R_{l}+1\right) F\left(k R_{l}+1\right) .
$$

Доказательство. Первые утверждения представляют собой несложные упражнения. Для доказательства остальных заметим, что если $M$ - наименьшее значение, при котором $(k, l) \in V_{R}(M)$, то $M-1<k R_{l} \leqslant M$ и

$$
N_{R}(k, l) \leqslant \operatorname{card} V_{R}(M)
$$

а в случае (ii)

$$
\operatorname{card} V_{R}(M) \leqslant \sum_{m=1}^{\infty} M R_{m}^{-1} \leqslant c\left(k R_{l}+1\right) .
$$

Аналогично, в случае (iii) выполняются неравенства

$$
\operatorname{card} V_{R}(M) \leqslant M \sum_{m=1}^{M} R_{m}^{-1} \leqslant\left(k R_{l}+1\right) F\left(k R_{l}+1\right) .
$$

1.6. Вероятностные распределения Л. Левина. Из неравенства (1.2) видно, что любую последовательность $\left\{R_{l}\right\}$, для которой сходится ряд $\sum_{l} R_{l}^{-1}$, можно использовать для построения биекции $\mathbb{Z}^{+} \times \mathbb{Z}^{+} \rightarrow \mathbb{Z}^{+},(k, l) \mapsto N_{R}(k, l)$, линейно растущей относительно $k$. Предположим, что эта биекция вычислима и, следовательно, может играть роль $\theta$ в доказательстве теоремы 1.4(b).

В этом случае для любого $l$ множество рациональных чисел $k / M$, удовлетворяющих условию $k / M \leqslant r_{l}:=R_{l}^{-1}$, должно быть разрешимым.

Даже если ослабить последнее условие, потребовав лишь перечислимости множества $\left\{(k, M, l) \mid k / M \leqslant r_{l}\right\}$ (в частности, потребовав, чтобы каждое число $r_{l}$ было вычислимо снизу), из сходимости ряда $\sum_{l} r_{l}$ следует, что для таких $r_{l}$ существует универсальная верхняя оценка (с точностью до константы). А именно, пусть $K P$ - экспонента от префиксной колмогоровской сложности на $\mathbb{Z}$, определенной с помощью некоторой оптимальной префиксной нумерации (подробности см. в [9], [2]).

1.6.1. Предложение [8]. Для любой последователъности чисел $r_{l} \geqslant 0$, для которой множество $\left\{(k, M, l) \mid k / M \leqslant r_{l}\right\}$ перечислимо и ряд $\sum_{l} r_{l}$ сходится, существует такая константа $c$, что $r_{l} \leqslant c \cdot K P(l)^{-1}$ для всех $l$.

Более общим образом, Л. Левин строит иерархию мер сложности, связанных с классом абстрактных норм, функционалов на последовательностях, вычислимых снизу.

Как объясняется во введении, идея данной статьи состоит в том, что рассмотрение этих математических законов распределения, возможно, приведет к новому объяснению статистических свойств некоторых наблюдаемых данных. 


\section{§2. Вычислимость, (про)перады и допустимые семейства}

2.1. Библиотеки, оракулы и операторы. В этом параграфе мы определяем допустимые множества $\Phi$ частичных $(m, n)$-функций, формализующие интуитивные понятия «библиотек программного обеспечения и их повторного использования» и «вычислений с оракулом». Колмогоровская сложность относительно такого множества является формализацией интуитивного понятия относительной сложности $K(f \mid g)$ в случаях, когда функции $f, g$ рекурсивны («повторное использование $g »$ ) и когда функция $g$ может быть невычислимой («вычисления с оракулом»).

Основными нашими объектами в этом параграфе являются не функиии, а объекты более сложных типов:

(i) программы для вычисления функиий, а впоследствии даже имена оракулов, сообщающих нам значения невычислимых функций, и программы, содержащие эти имена;

(ii) операторы, т. е. программы, вычисляющие некоторые функиии, аргументы и значения которых сами являются программами.

Причина для подобного смещения внимания состоит в следующем. Уже множество частично рекурсивных $(m, n)$-функций при $m \geqslant 1$, как и его расширения, с которыми мы имеем дело в данной статье, не является конструктивным миром. Напротив, множество программ на данном языке программирования, вычисляющих рекурсивные функции, например множество машин Тьюринга или текстов в лямбда-исчислении, является конструктивным, но снабжено невъчислимым отношением эквивалентности «две программы вычисляют одну и my же функиию». Подобные миры мы будем называть «методами программирования».

Опишем ситуацию в более точных терминах. Пусть $X, Y$ - два конструктивных мира. Метод программирования - это конструктивный мир $P(X, Y)$, заданный вместе с отображением $P(X, Y) \rightarrow \operatorname{ParSet}(X, Y), p \mapsto \bar{p}$, где ParSet категория множеств с частичными отображениями в качестве морфизмов. Имя программы (скажем, $p)$, снабженное чертой, будет всегда обозначать функцию $\bar{p}: X \rightarrow Y$, которую она вычисляет.

Хороший метод программирования должен обладать дополнительными свойствами согласованности. Для краткости будем рассматривать только бесконечные конструктивные миры и предположим, что метод программирования $P$ вычисляет все рекурсивные изоморфизмы. Тогда мы можем продолжить его на любые два бесконечных конструктивных мира, и при этом естественно потребовать существования по крайней мере двух дополнительных программ/операторов

$$
\begin{aligned}
& E v \in P(X \times P(X, Y), Y), \quad \overline{E v}(x, p):=\bar{p}(x) \quad \text { при } x \in X, p \in P(X, Y), \\
& \text { Comp: } P(P(X, Y) \times P(Y, Z)) \rightarrow P(X, Z), \quad \overline{\overline{\operatorname{Comp}}(f, g)}=\bar{g} \circ \bar{f} \text {. }
\end{aligned}
$$

Такие объекты мы будем называть $E v$ - и Comp-операторами; мы будем говорить, что они являются поднятиями соответствующих операций над функциями: взятия значения в точке и композиции.

Более подробное описание математического контекста см. в [13, гл. IX, разд. $3-5]$. 
В дальнейшем, начав с некоторого метода программирования $P$, вычисляющего по крайней мере все частично рекурсивные $(m, n)$-функции, мы опишем способы его продолжения, необходимые для формализации понятий повторного использования библиотек и вычисления с оракулами.

2.2. Построение допустимых множеств. Каждое такое множество $\Phi$ будет определяться следующим образом.

(i) Выберем конструктивный мир $S$, состоящий из программ для вычисления $(m, n)$-функций. Он будет объединением двух частей: (программ для вычисления) элементарных функций и библиотечных функиий. Все элементарные функции будут вычислимы (по Тьюрингу), т.е. (частично) рекурсивны. Библиотечные функции должны составлять конструктивный мир (возможно, конечный) с некоторой фиксированной нумерацией. Номер библиотечной программы называется ее адресом. И библиотечные, и элементарные функции будут образовывать разрешимые подмножества в $S$.

(ii) Определим множество операторов, которые можно применять к конечным строкам частичных $\left(m_{i}, n_{i}\right)$-функций. Все такие операторы будут получаться итерациями нескольких основных операторов, таких, как упомянутые выше операторы Comp и $E v$. Мир OP программ/имен таких операторов легко кодируется некоторым конструктивным миром помеченных графов.

(iii) Возьмем элемент $P \in O P$ и укажем конечную строку $s:=\left(f_{1}, \ldots, f_{r}\right)$ (адресов) функций из $S$, которую $P$ может принимать на входе. Пара $(P, s)$ является программой для вычисления конкретной строки $(m, n)$-функций.

(iv) Наконец, $\Phi$ будет определяться как минимальное множество функций, вычислимых с помощью программ из $S$, замкнутое относительно применения всех операторов из $O P$.

Теперь мы приведем наши основные примеры объектов, неформально описанных в пп. (i)-(iv).

2.3. Язык ориентированных графов. В нашем мире $O P$ каждый оператор $\rho$ принимает на входе конечную последовательность частичных функций $f_{i}:\left(\mathbb{Z}^{+}\right)^{m_{i}} \rightarrow\left(\mathbb{Z}^{+}\right)^{n_{i}}, m_{i}, n_{i} \geqslant 0, i=1, \ldots, k$, и преобразует ее в другую конечную последовательность частичных функций $g_{i}:\left(\mathbb{Z}^{+}\right)^{p_{i}} \rightarrow\left(\mathbb{Z}^{+}\right)^{q_{i}}, m_{i}, n_{i} \geqslant 0$, $i=1, \ldots, l$. Сигнатурой оператора $\rho$ мы будем называть семейство

$$
\operatorname{sign}(\rho):=\left[\left(m_{1}, n_{1}\right), \ldots,\left(m_{k}, n_{k}\right) ;\left(p_{1}, q_{1}\right), \ldots,\left(p_{l}, q_{l}\right)\right] .
$$

Если сигнатуры двух операторов $\rho, \sigma$ согласованы таким образом, что оператор $\rho$ принимает на входе выход оператора $\sigma$, то из них можно составить композицию $\rho \circ \sigma$.

Ниже мы явно опишем множество $O P_{0}$ основных операторов, после чего определим $O P$ как минимальное множество операторов, содержащее $O P_{0}$ и замкнутое относительно композиции.

Наглядное изображение элементов множества $O P$ дают помеченные ориентированные графы (точнее, их классы изоморфизма); формальные определения см. в $[1, \S 1]$.

Более точно, каждый основной оператор $\rho$ с сигнатурой (2.3) представляется венчиком - графом, имеющим одну вершину с меткой $\rho, k$ флагов, направленных к этой вершине (входов), и $l$ флагов, направленных от этой вершины (выходов). Кроме того, входы и выходы должны быть вполне упорядочены и помечены соответствующими парами $\left(m_{i}, n_{i}\right)$. 
Помеченные ориентированные графы с более чем одной вершиной получаются из дизъюнктных конечных семейств венчиков пересаживанием некоторых выходов на некоторые входы. Пара (выход, вход) для пересаживания должна иметь одинаковые метки $(m, n)$. Непересаженные флаги образуют входы/выходы нового графа.

Заметим, что в ориентированных графах не допускаются ориентированные петли (см. [1, п. 1.3.2]).

2.4. Основные операторы. В этом разделе мы опишем операции над строками функций, которые должны быть представлены соответствующими операторами. Для краткости мы будем обозначать эти операторы единичными греческими буквами.

(a) Композииия функиии. Этот основной оператор (скажем, $\gamma$ ) имеет сигнатуру вида $[(m, n),(n, q) ;(m, q)]$. Из двух частичных функций $f:\left(\mathbb{Z}^{+}\right)^{m} \rightarrow\left(\mathbb{Z}^{+}\right)^{n}$ и $g:\left(\mathbb{Z}^{+}\right)^{n} \rightarrow\left(\mathbb{Z}^{+}\right)^{q}$ он составляет их композицию $g \circ f:\left(\mathbb{Z}^{+}\right)^{m} \rightarrow\left(\mathbb{Z}^{+}\right)^{q}$. Напомним, что $D(g \circ f):=f^{-1}(D(g))$. Это частный случай оператора Comp.

(b) Соединение. Этот основной оператор (скажем, $\sigma$ ) имеет сигнатуру вида $\left[\left(m, n_{1}\right), \ldots,\left(m, n_{k}\right) ;\left(m, n_{1}+\cdots+n_{k}\right)\right]$. Из $k$ частичных функций $f_{i}:\left(\mathbb{Z}^{+}\right)^{m} \rightarrow$ $\left(\mathbb{Z}^{+}\right)^{n_{i}}$ он составляет функцию $\left(f_{1}, \ldots, f_{k}\right)$ :

$$
\begin{gathered}
D\left(\left(f_{1}, \ldots, f_{k}\right)\right)=D\left(f_{1}\right) \cap \cdots \cap D\left(f_{k}\right), \\
\left(f_{1}, \ldots, f_{k}\right)\left(x_{1}, \ldots, x_{m}\right)=\left(f_{1}\left(x_{1}, \ldots, x_{m}\right), \ldots, f_{k}\left(x_{1}, \ldots, x_{m}\right)\right) .
\end{gathered}
$$

(c) Рекурсия. Этот основной оператор (скажем, $\rho$ ) имеет сигнатуру вида $[(m, 1),(m+2,1) ;(m+1,1)]$. Из частичных функций $f:\left(\mathbb{Z}^{+}\right)^{m} \rightarrow \mathbb{Z}^{+}$и $g:\left(\mathbb{Z}^{+}\right)^{m+2}$ $\rightarrow \mathbb{Z}^{+}$он составляет такую функцию $h:\left(\mathbb{Z}^{+}\right)^{m+1} \rightarrow \mathbb{Z}^{+}$, что

$$
\begin{gathered}
h\left(x_{1}, \ldots, x_{m}, 1\right)=f\left(x_{1}, \ldots, x_{m}\right), \\
\left.h\left(x_{1}, \ldots, x_{m}, k+1\right)=g\left(x_{1}, \ldots, x_{m}, k, h\left(x_{1}, \ldots, x_{m}, k\right)\right)\right)
\end{gathered}
$$

при $k \geqslant 1$.

Область определения $D(h)$ функции $h$ также определяется рекурсивно:

$$
\begin{array}{r}
\left(x_{1}, \ldots, x_{m}, 1\right) \in D(h) \Longleftrightarrow\left(x_{1}, \ldots, x_{m}\right) \in D(f) \text { и }\left(x_{1}, \ldots, x_{m}, k+1\right) \in D(h) \\
\Longleftrightarrow\left(x_{1}, \ldots, x_{m}, k\right) \in D(h) \text { и }\left(x_{1}, \ldots, x_{m}, k, h\left(x_{1}, \ldots, x_{m}, k\right)\right) \in D(g)
\end{array}
$$

при $k \geqslant 1$.

(d) Onератор $\mu$. Этот оператор имеет сигнатуру $[(n+1,1) ;(n, 1)]$. Данную $(n+1,1)$-функцию $f$ он преобразует в $(n, 1)$-функцию $h$ с областью определения

$$
\begin{aligned}
& D(h)=\left\{\left(x_{1}, \ldots, x_{n}\right) \mid \text { существует } x_{n+1} \geqslant 1,\right. \text { такое, что } \\
& \left.\quad f\left(x_{1}, \ldots, x_{n}, x_{n+1}\right)=1 \text { и }\left(x_{1}, \ldots, k\right) \in D(f) \text { для всех } k \leqslant x_{n+1}\right\} .
\end{aligned}
$$

На этой области определения

$$
h\left(x_{1}, \ldots, x_{n}\right)=\min \left\{x_{n+1} \mid f\left(x_{1}, \ldots, x_{n+1}\right)=1\right\} .
$$

(е) Тождественная операция $\iota$.

2.5. Конструктивный мир операций $\boldsymbol{O P}$. Возвращаясь к разд. 2.2, мы определяем $O P$ как множество конечных помеченных ориентированных графов с вполне упорядоченными множествами входов/выходов в каждой вершине, 
удовлетворяющих следующему условию: каждая вершина графа помечена одной из букв $\gamma, \sigma, \rho, \mu, \iota$, а ее входы/выходы помечены компонентами соответствующей сигнатуры.

Выбрав любой стандартный способ кодировки таких графов строками над фиксированным конечным алфавитом, мы можем определить структурную нумерацию множества $O P$, упорядочив эти слова в алфавитном порядке. Подмножество правильных строк, кодирующих графы, должно быть разрешимым подмножеством в множестве всех строк, а все естественные функции, такие, как

граф $\mapsto$ последовательность всех входов с их $(m, n)$-метками,

должны быть общерекурсивны.

В итоге, каждый элемент множества $O P$ задает операцию над конечными множествами частичных функций, результатом которой является другое конечное множество частичных функций. Более того, $O P$ можно расширить до свободной (про)перады, действующей на конечных строках частичных функций. Если сигнатура строки не соответствует сигнатуре входов операции, мы можем и будем считать, что результатом операции является пустая функция. Однако подобное расширение требует некоторой осторожности и использования высших категорных конструкций.

2.6. Основные частичные функции. Пусть $S$ - конструктивный мир программ/оракулов, вычисляющих частичные $(m, n)$-функции. Обозначим через $O P(S)$ минимальное множество таких программ, содержащее $S$ и замкнутое относительно применения к ним операторов из $O P$. В оставшейся части статьи мы всегда включаем в $S$ множество основных рекурсивных функций $S_{r e c}$, такое, что $O P\left(S_{r e c}\right)$ состоит из всех (частично) рекурсивных функций. В $[13$, гл. $\mathrm{V}$, разд. 2] в качестве $S_{r e c}$ выбрано множество, состоящее из следующих функций:

$$
\begin{gathered}
\text { suc: } \mathbb{Z}^{+} \rightarrow \mathbb{Z}^{+}, \quad x \mapsto x+1, \\
1^{(n)}:\left(\mathbb{Z}^{+}\right)^{m} \rightarrow \mathbb{Z}^{+}, \quad\left(x_{1}, \ldots, x_{m}\right) \mapsto 1, n \geqslant 0 . \\
\operatorname{pr}_{i}^{m}:\left(\mathbb{Z}^{+}\right)^{m} \rightarrow \mathbb{Z}^{+}, \quad\left(x_{1}, \ldots, x_{m}\right) \mapsto x_{i}, n \geqslant 1 .
\end{gathered}
$$

2.7. Допустимые множества и повторное использование библиотек. Стандартная колмогоровская сложность частично рекурсивных функций определяется относительно допустимого множества $\Phi$ функций, вычислимых с помощью программ из $O P\left(S_{r e c}\right)$. Если включить в множество $S$ лишь некоторые программы для рекурсивных функций, то полное множество вычислимых функций $\Phi$ не увеличится, но некоторые функции будут вычисляться гораздо более короткими программами, поскольку ценой написания программы для библиотечной программы можно пренебречь.

\section{8. Допустимые множества, включающие оракулов для невычис-} лимых функций. Здесь возникает еще одно затруднение: в мире $O P(S)$ требование (ii) из нашего определения допустимых множеств функций не выполняется автоматически, как это было в случае, когда соответствующее множество состояло только из рекурсивных функций.

Чтобы преодолеть его, следует добавить в список основных операторов оператор $E v$, заданный формулой (2.1). Не существует очевидного способа описать участие этого оператора в итерации наших прежних основных операций на языке помеченных графов; поэтому здесь требуется более систематический подход: 
по всей видимости, мы находимся в царстве «расширяющейся конструктивной вселенной» (некоторая популяризация этого понятия содержится в [13, гл. IX, разд. 3].

\section{ЛитератУРА}

[1] D. Borisov, Yu. Manin, Generalized operads and their inner cohomomorphisms, in: Geometry and Dynamics of Groups and Spaces (In memory of Aleksader Reznikov), Prog. Math., vol. 265 (eds. M. Kapranov et al.), Birkhäuser, Basel, 2008, 247-308; http://arxiv.org/abs/math/0609748.

[2] C. S. Calude, L. Staiger, On universal computably enumerable prefix codes, Math. Structures Comput. Sci., 19:1 (2009), 45-57.

[3] S. Dehaene, The Number Sense. How the Mind Creates Mathematics, Oxford Univ. Press, New York, 1997.

[4] S. Dehaene, J. Mehler, Cross-linguistic regularities in the frequency of number words, Cognition, 43:1 (1992), 1-29.

[5] J.-P. Delahaye, Les entiers ne naissent pas égaux, Pour la Science, $\mathrm{n}^{\circ} 421$ (Nov. 2012), 80-85.

[6] K. M. Frahm, A. D. Chepelianskii, D. L. Shepelyansky, PageRank of integers, http://arxiv.org/abs/1205.6343.

[7] Shi-Ming Huang, David C. Yen, Luen-Wei Yang, Jing-Shiuan Hua, An investigation of Zipf's Law for fraud detection, Decision Support Systems, 46:1 (2008), 70-83.

[8] Л. А. Левин, О различных мерах сложности конечных объектов (аксиоматическое описание), Докл. АН СССР, 227:4 (1976), 804-807.

[9] Ming Li, P. Vitányi, An introduction to Kolmogorov complexity and its applications, Springer-Verlag, New York, 1993.

[10] B. Mandelbrot, An information theory of the statistical structure of languages, in: Communication Theory, Proc. of the Symposium on Application of Communicaions Theory, Butterworth, Woburn, MA, 1953, 486-502.

[11] D. Yu. Manin, Zipf's Law and avoidance of excessive synonymy, Cognitive Science, 32:7 (2008), 1075-1078; http://arxiv.org/abs/0710.0105.

[12] D. Yu. Manin, Mandelbrot's model for Zipf's Law. Can Mandelbrot's model explain Zipf's Law for language?, J. Quantitative Linguistics, 16:3 (2009), 274-285.

[13] Yu. I. Manin, A Course in Mathematical Logic for Mathematicians. 2nd ed., Graduate Texts in Mathematics, vol. 53, Springer-Verlag, 2010.

[14] Yu. Manin, Renormalization and computation II, Math. Struct. Comput. Sci., 22:5 (2012), 729-751; http://arxiv.org/abs/0908.3430.

[15] Yu. Manin, Kolmogorov complexity as a hidden factor of scientific discourse: from Newton's law to data mining, Talk at the Plenary Session of the Pontifical Academy of Sciences on "Complexity and Analogy in Science: Theoretical, Methodological and Epistemological Aspects", Vatican, November 5-7, 2012; http://arxiv.org/abs/1301.0081.

[16] Yu. Manin, M. Marcolli, Kolmogorov complexity and the asymptotic bound for errorcorrecting codes, http://arxiv.org/abs/1203.0653.

[17] B. C. Murtra, R. Solé, On the universality of Zipf's Law, Santa Fe Institute, 2010 (доступно в Интернете).

[18] A. Nabutovsky, S. Weinberger, The fractal nature of Riemm/Diff. I, Geometriae Dedicata, 101 (2003), 1-54.

[19] H. Rogers, Gödel numberings of partial recursive functions, J. Symb. Logic, 23 (1958), $331-341$.

[20] C. P. Schnorr, Optimal enumerations and optimal Gödel numberings, Math. Systems Theory, 8:2 (1974), 182-191. 
[21] T. Tao, E pluribus unum: From Complexity, Universality. Daedalus, J. Amer. Acad. Sci. (Summer 2012), 23-34.

[22] Todd L. Veldhuizen, Software Libraries and Their Reuse: Entropy, Kolmogorov Complexity, and Zipf's Law, http://arxiv.org/abs/cs/0508023.

[23] N. S. Yanofsky, Towards a definition of an algorithm, J. Logic Comput., 21:2 (2011), 253-286; http://arxiv.org/abs/math/0602053.

[24] G. K. Zipf, The Psycho-Biology of Language, Routledge, London, 1936.

[25] G. K. Zipf, Human Behavior and the Principle of Least Effort, Addison-Wesley, Reading, MA, 1949.

[26] А. К. Звонкин, Л. А. Левин, Сложность конечных объектов и обоснование понятий информации и случайности с помощъю теории алгоритмов, УМН, 25:6(156) (1970), 85-127.

Max-Planck-Institut für Mathematik, Bonn, Germany e-mail: manin@mpim-bonn.mpg.de 